\title{
Simulation studies of the laser ablation ion source at the SHIPTRAP setup
}

\section{Brankica Anđelić1,2 (D . Michael Block ${ }^{2,3,4} \cdot$ Premaditya Chhetri ${ }^{2,3} \cdot$ Julia Even $^{1}$. Francesca Giacoppo ${ }^{2,3}$. Nasser Kalantar-Nayestanaki ${ }^{1}$. Oliver Kaleja ${ }^{3,4,5}$. Tobias Murböck ${ }^{2,3} \cdot$ Fabian Schneider ${ }^{2,4}$ • Sebastian Raeder ${ }^{2,3}$}

Published online: 18 March 2020

(C) The Author(s) 2020

\begin{abstract}
A gas-filled miniature Radio-Frequency Quadrupole (mini-RFQ) was recently implemented into the SHIPTRAP laser ablation ion source to thermalize the laser-ablated ions and thus improve production efficiency as well as sample preparation. This source provides reference ions of various elements for online experiments with the SHIPTRAP mass spectrometer. In addition, it can be used to provide long-lived rare and radioactive isotopes available only in small sample sizes for high-precision mass measurements or to study systematic uncertainties. The performance of the laser ablation ion source was simulated using the COMSOL Multiphysics modeling software package. These studies indicate that a revised mechanical geometry and an optimized RF field improve the performance significantly.
\end{abstract}

Keywords Penning traps $\cdot$ Mass spectrometry $\cdot$ Laser ablation ion source $\cdot$ Surface ionization ion source

\section{Introduction}

In several areas of physics, high-precision measurements of atomic masses and mass differences, so called $Q$-values, are required. Penning-trap mass spectrometry (PTMS) is the most precise and accurate method for mass measurements of stable and short-lived

This article is part of the Topical Collection on Proceedings of PLATAN 2019, 1st International Conference, Merger of the Poznan Meeting on Lasers and Trapping Devices in Atomic Nuclei Research and the International Conference on Laser Probing, Mainz, Germany 19-24 May 2019

Edited by Krassimira Marinova, Michael Block, Klaus D.A. Wendt and Magdalena Kowalska

$\checkmark$ Brankica Anđelić

b.andelic@rug.nl

1 KVI-CART, University of Groningen, Groningen, 9747 AA, The Netherlands

2 Helmholtz-Institut Mainz, Mainz, 55128, Germany

3 GSI Helmholtzzentrum für Schwerionenforschung Darmstadt, Darmstadt, 64291, Germany

4 Johannes-Gutenberg Universität, Mainz, 55099, Germany

5 Max-Planck-Institut für Kernphysik Heidelberg, Heidelberg, 69117, Germany 
nuclides [1]. The SHIPTRAP spectrometer [2] located at the GSI Helmholtzzentrum für Schwerionenforschung Darmstadt, Germany, is one of the Penning trap-based facilities that performs direct high-precision mass measurements of isotopes produced on-line in fusion-evaporation reactions as well as delivered from off-line ion sources. Aside from the experiments investigating heavy and super-heavy elements [3, 4], the physics program of SHIPTRAP comprises the very neutron-deficient nuclides below tin [5, 6] as well as high-precision measurements of $Q$-values contributing to neutrino physics [7].

According to the principles of PTMS, an ion's mass-to-charge ratio, $m / q$, is determined through a direct measurement of the ion cyclotron frequency $\omega_{c}$ in a strong and homogeneous magnetic field $B$ :

$$
\omega_{c}=\frac{q}{m} B .
$$

In order to calibrate accurately the strength of the magnetic field in a Penning trap, it is necessary to perform reference measurements of an ion with well-known mass $m_{\text {ref. Even- }}$ tually, the mass of the atom under investigation is determined from the experimental result for the frequency ratio of its ion:

$$
m=\frac{q}{q_{\mathrm{ref}}} \frac{\omega_{c, \mathrm{ref}}}{\omega_{c}}\left(m_{\mathrm{ref}}-q_{\mathrm{ref}} \cdot m_{e}\right)+q \cdot m_{e}
$$

where $q_{\text {ref }}$ is the charge state of the reference ion and $m_{e}$ is the electron mass.

In order to minimize systematic frequency shifts, the mass-to-charge ratio of the reference ions and the ions of interest have to be similar [8]. The SHIPTRAP setup is equipped with a surface ion source as well as with a laser-ablation ion source providing reference ions of various elements and with different mass-to-charge ratios. Thanks to these installations, ions can be produced at stable count rates for long measurement times and for studies of systematic uncertainties. In particular, the laser ablation ion source has allowed many offline measurements with stable and long-lived ions as, for instance, to measure the electron capture $Q$-value of ${ }^{163} \mathrm{Ho}$ with a sub-ppb uncertainty (see Ref. [7]), using a sample of about $10^{16}$ atoms.

To further boost the performance of the laser ion source and to enable measurements of long-lived rare and radioactive isotopes available in sample size as low as $10^{14}$ atoms, further improvements of the current laser ablation ion source in terms of production efficiency and sample preparation are required. In this work, the description of of a new-designed laser ablation ion source is given along with simulation studies.

\section{Experimental setup}

The SHIPTRAP setup (see Fig. 1) is located behind the velocity filter SHIP [9], which separates fusion-evaporation products with kinetic energies of several tens of $\mathrm{MeV}$ from the faster primary beam. In order to prepare the reaction products for Penning trap mass measurements, the ions are slowed down and thermalized in collisions with helium gas inside a buffer-gas stopping cell operated at cryogenic temperature (usually $40 \mathrm{~K}$ ) and at as pressure around $7.5 \mathrm{mbar}$, depending on the initial energy of the fusion-evaporation products. More details on the operation and the recent optimizations of the cryogenic cell can be found in Ref. [10]. By using a combination of electrostatic and radio-frequency fields in addition to the gas-flow, the ions are extracted from the gas cell into a radio-frequency quadrupole (RFQ) section at low-vacuum $\left(\sim 10^{-2} \mathrm{mbar}\right)$ and then guided towards the RFQ buncher. In this region, through collisions with ultra-pure He buffer-gas at a pressure of about $10^{-3} \mathrm{mbar}$, the ions are cooled, collected and transmitted in bunches. Thereafter, 


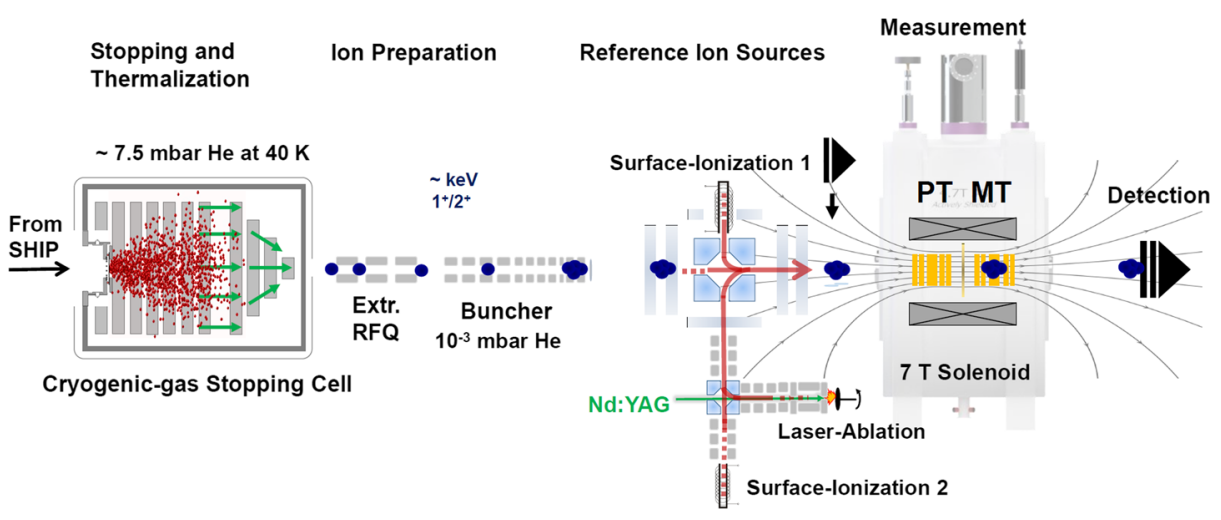

Fig. 1 Schematic view of the SHIPTRAP setup and its major components (modified from Ref. [10]): the cryogenic-gas stopping cell, the extraction RFQ and the buncher for ion preparation, the reference ion sources and the Penning traps. The triangles indicate detector positions

single ion bunches are injected into the double Penning-trap system located inside a $7 \mathrm{~T}$ superconducting magnet. The first trap is called purification trap (PT) and allows isobaric purification of the species of interest with a mass resolving power of about 100,000 using the side-band cooling technique [11]. The ions of interest are then transferred into the measurement trap (MT) where their radial motion is excited. Finally, the ions are ejected from the MT to a position-sensitive detector where the ion motion is projected to determine the cyclotron frequency. The employed Phase-Imaging Ion-Cyclotron-Resonance (PI-ICR) technique has been developed at SHIPTRAP recently [12].

At present, there are three reference ion sources: a laser ablation ion source and two surface ion sources (see Fig. 1). These are installed in separate beamlines connected to the main beamline by an electrostatic quadrupole bender. By using this bender the ions are guided towards the traps. A second surface ion source is installed recently as replacement for surface ion source 1, which will be removed in order to make a connection to a second beamline branch where a single-ion mass spectrometry is currently under development.

\subsection{Reference ion sources}

The section that contains the reference ion sources is schematically presented in Fig. 2. Within the surface ion source, the ions of ${ }^{39} \mathrm{~K},{ }^{85} \mathrm{Rb}$ and ${ }^{133} \mathrm{Cs}$ are produced by heating the source, with an electric current leading to emission of positive ions and neutrals. Since the ions are emitted continuously, in order to chop the ion beam the electrostatic voltages of plate 1 and 2 are switched between positive and negative values such that the ions are either repelled back or transferred through the lenses and the deflector towards the beamline.

The second branch in Fig. 2 shows another type of ion source that is based on the laser ablation process. When a solid target is irradiated with focused, high-power laser pulses, a plasma is created from which positive ions of the surface material can be extracted. The ion yield in the laser-ablation process depends on the laser wavelength and pulse length, as well as on the laser pulse energy. The laser ion source installed at SHIPTRAP, shown in Fig. 2, 


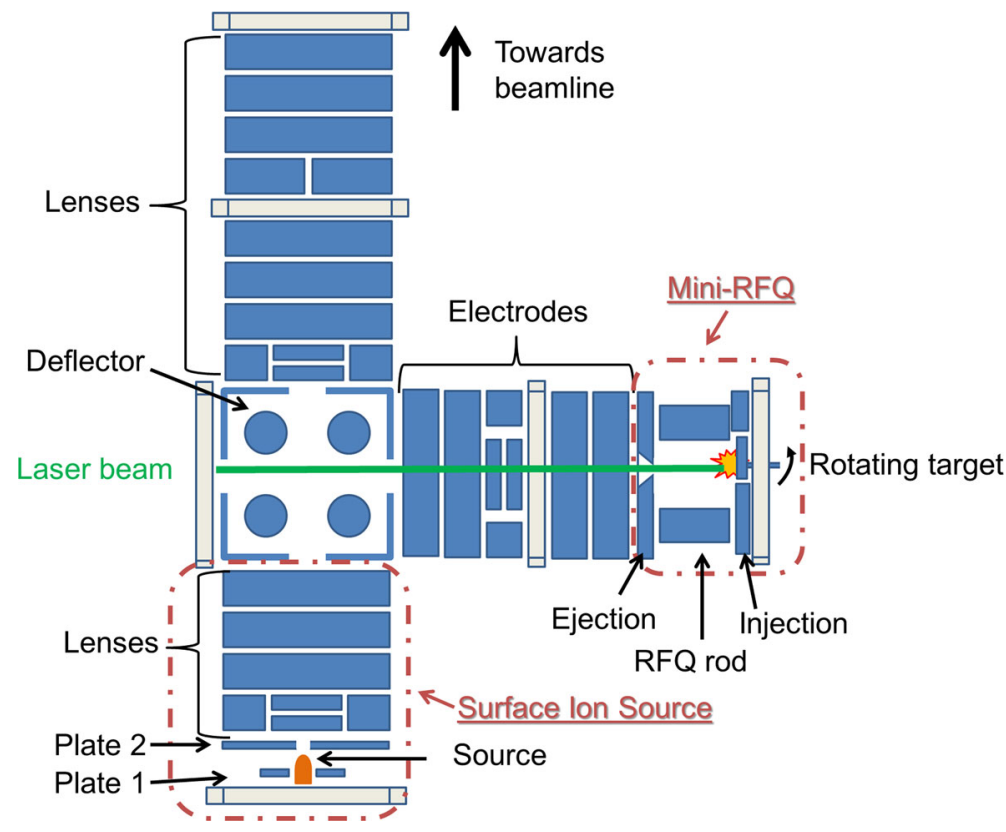

Fig. 2 The layout of the surface ion source 2 and the laser ablation ion source which contains the mini-RFQ section

exploits a frequency-doubled Nd:YAG laser at $532 \mathrm{~nm}$ (Continuum, Minilite series) to irradiate a small target sample and produce ions. The energy of the laser pulse is adjustable up to $25 \mathrm{~mJ}$, while the pulse length is $5 \mathrm{~ns}$ at a maximum repetition rate of $15 \mathrm{~Hz}$. In order to increase the power density on the target, the laser beam is focused using optical lenses. The target usually consists of a thin metallic foil or a liquid-solution deposited and dried on a titanium supporting foil. The target holder can accommodate multiple samples and is driven by a step motor that allows fine rotation in order to scan the position of the same sample as well as to access another target. Once ablated from the surface of the sample, the ions are injected into a mini-RFQ [13], which consists of an injection electrode, four parallel rods and an ejection electrode. Here, it is possible to confine the ions axially by applying electrostatic voltages to the injection electrode, the rods and the ejection electrode in a way that a potential minimum is created in the mini-RFQ volume. The radial confinement is achieved by applying a radio-frequency (RF) voltage with a frequency of about $500 \mathrm{kHz}$ to the rods such that each rod has a $\pi$ phase difference with respect to the adjacent one. Through the collisions with helium buffer-gas atoms at a pressure around $0.01 \mathrm{mbar}$, the ions are thermalized and accumulated in the potential minimum inside the RFQ structure. By lowering the bias potential of the ejection electrode, a bunch of cooled ions is ejected, accelerated and guided by an electrostatic gradient generated by the following electrodes towards the deflector in a high-vacuum regime. The bunch of ions is here deflected and guided through electrostatic lenses to the main section of the beamline. As shown in Fig. 2, some of the electrostatic electrodes and neighboring lenses are segmented and help to correct the ion trajectory while the others are usually used as an Einzel lens to focus the ions. 


\section{a) DC potential}

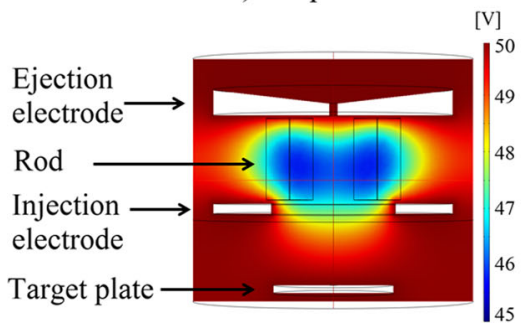

b) DC potential

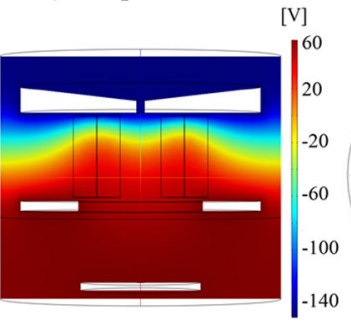

c) DC and RF potential

[V]

100

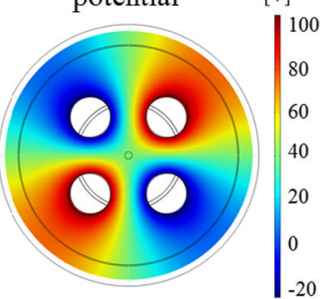

Fig. 3 Mini-RFQ simulations of the electric potential using the COMSOL Multiphysics software: a DC potential distribution during the injection and the cooling process shown in longitudinal plane, $\mathbf{b}$ DC potential distribution during the ejection process shown in longitudinal plane, and $\mathbf{c}$ DC and RF potential distribution shown together in radial plane

\section{Laser ablation ion source}

In order to increase the sensitivity for experiments on rare isotopes, the laser-ablated ions have to be produced, transported and injected into the PT with maximum efficiency and minimum energy and time spread. This requires that the ions are captured and cooled right after the ablation process. To thermalize the laser-ablated ions and to collect them in a bunch, we have implemented a mini-RFQ with a similar design as the one used at the mass spectrometer TRIGA-TRAP [13]. As the name mini-RFQ suggests, it is a very compact device: the four rods are $20 \mathrm{~mm}$ long with a diameter $11.5 \mathrm{~mm}$ and a rod spacing of $10 \mathrm{~mm}$. The typical operating parameters to confine ions with a mass to charge ratio of about 200 are $U_{D C}=55 \mathrm{~V}$ and the amplitude of the RF field is $U_{R F}=65 \mathrm{~V}$ at a frequency of $500 \mathrm{kHz}$. The quadrupole rods are placed in between the injection and the ejection electrode. The injection electrode contains a circular cutout the center of which is slightly offset from the center axis of the quadrupole, while the ejection electrode has a conical shape with an initial diameter of $2 \mathrm{~mm}$. Depending on the voltage applied to these electrodes, the ions are either injected and trapped by an up to $10 \mathrm{~V}$ potential well or ejected from the mini-RFQ by increasing the Direct Current (DC) potential of the injection and lowering the potential of the ejection electrode. The ablation target is mounted at $17 \mathrm{~mm}$ distance below the injection electrode and they are kept at the same potential.

a)

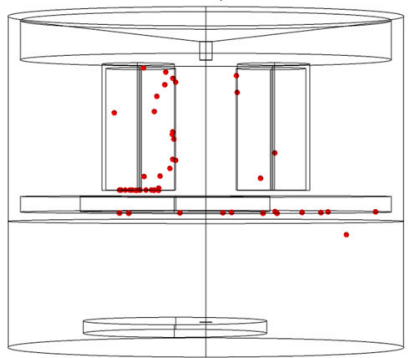

b)

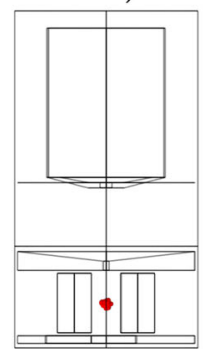

c)

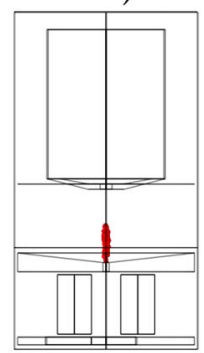

d)

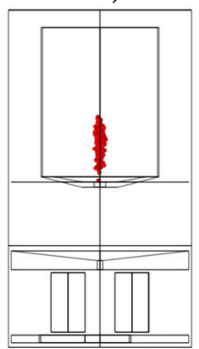

Fig. 4 Positions of ions extracted from the ion trajectory simulations considering: a non optimized conditions, $\mathbf{b}$ adjusted geometry and increased RF amplitude at the end of cooling process, $\mathbf{c}$ adjusted geometry and increased RF amplitude during ejection from the mini-RFQ, and $\mathbf{d}$ adjusted geometry and increased RF amplitude during extraction after the inner canal of the first electrode 

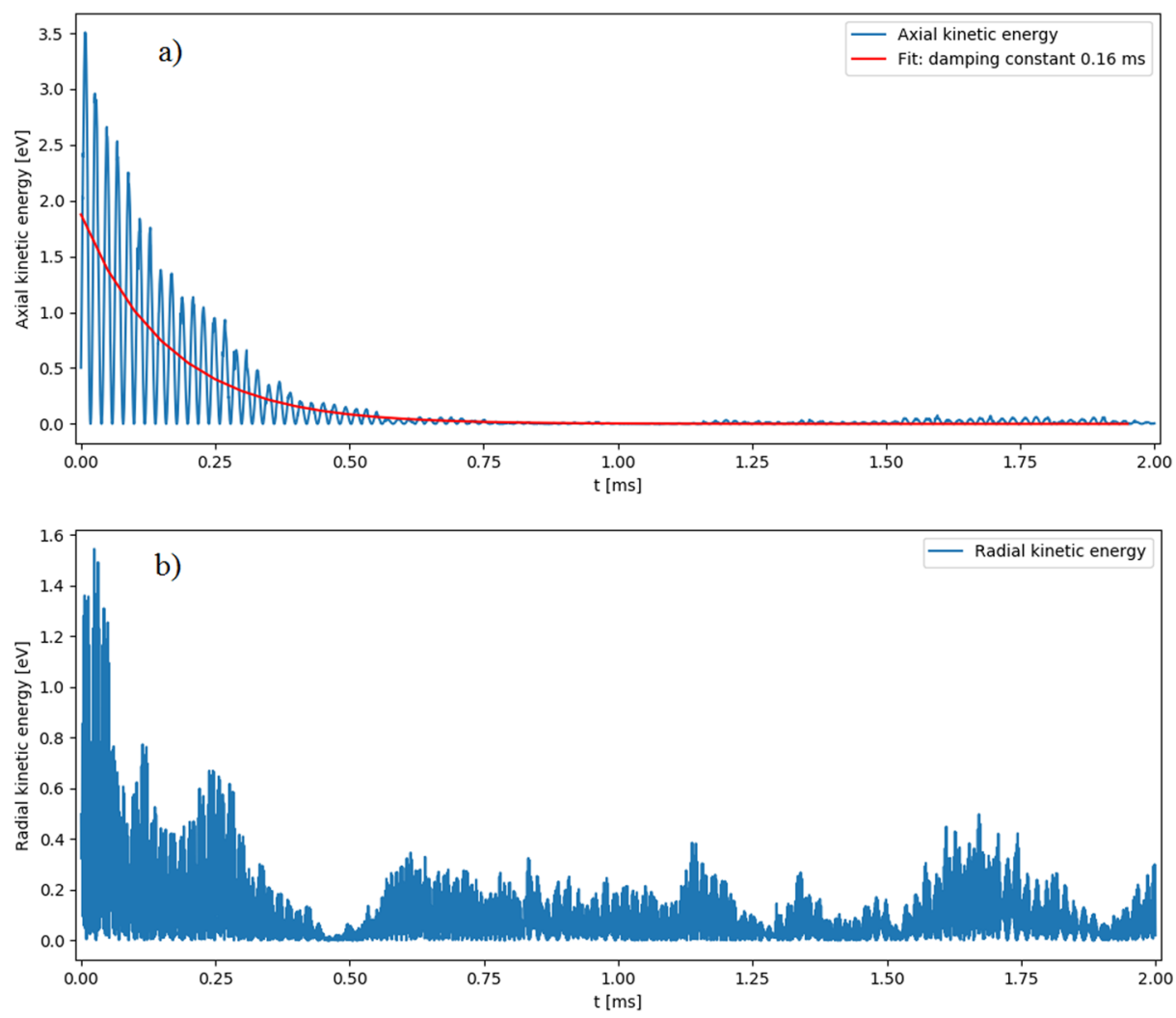

Fig. 5 The axial (a) and the radial (b) kinetic energy of a trapped ion in the Mini-RFQ. The red line represents an exponential fit to the simulated data shown in blue color

Aiming at improving the overall efficiency of the laser ablation ion source, we performed simulation studies of the mini-RFQ in the described configuration using the COMSOL Multiphysics modeling software [14]. The goal was to optimize ion-bunch production and transmission, as well as the operational parameters which have a major influence on the efficient injection into the PT, such as the energy spread of the bunch, which should be smaller than few $\mathrm{eV}$ and its time distribution in the order of few microseconds.

In the simulations, 200 singly-charged ions with a mass of $193 \mathrm{u}$, where $\mathrm{u}$ is the unified atomic mass unit, and an initial kinetic energy of $1 \mathrm{eV}$ were used. We assume that these ions are ablated out of the target randomly within a cone with an opening angle of $90^{\circ}$. Figure 3a shows a mapping of the DC potential distribution during the injection of the ions into the mini-RFQ and during the cooling process, while Fig. $3 \mathrm{~b}$ shows the DC potential during ejection of the ions from the mini-RFQ. The distribution of the RF potential, which confines the ions radially, together with the DC potential distribution is shown in Fig. $3 \mathrm{c}$ in radial plane.

The ion motion is simulated using hard-sphere collisions with helium gas at a pressure of 0.01 mbar. The collision cross section is calculated from the van der Waals radii of the ion and the helium atom. However, once the ion energy approaches thermal velocities, the viscous drag model gives a more appropriate description of the ion motion. A sharp line 
a) Electric potential

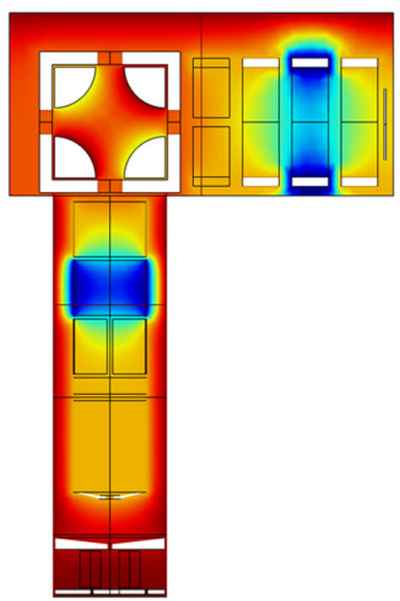

$[\mathrm{kV}]$

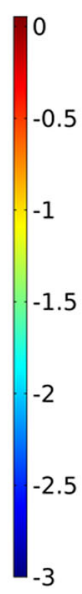

b) Ion trajectories

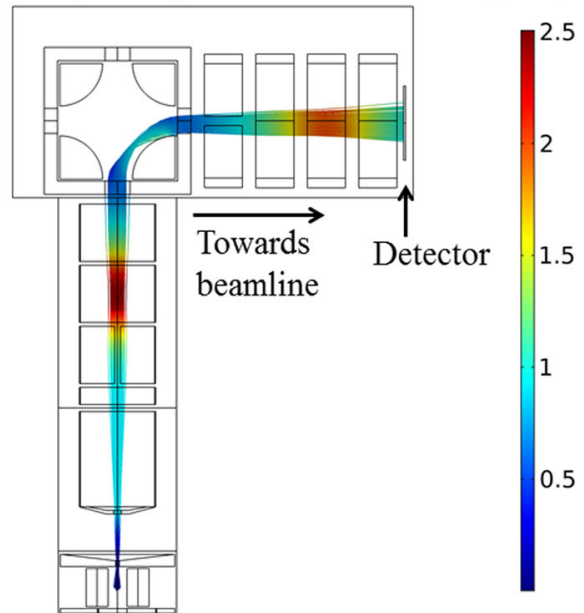

Fig. 6 Extended simulation: a the DC potential distribution during extraction and transportation and $\mathbf{b}$ the ion trajectories showing extraction and transportation to the main beamline

separating the validity of the two models cannot be clearly defined and as, in terms of ion losses, the ion bunch preparation for an efficient injection into the Penning trap system is more sensitive to the injection and the ejection from the mini-RFQ than to the cooling process (assuming that after a few milliseconds the ions reach thermal equilibrium), we decided to use the hard-sphere collisions model. The final ion positions after a storage time of $2 \mathrm{~ms}$ are shown in Fig. 4a. In the simulation, we observed strong losses mostly during the ion injection due to the poor geometrical acceptance of the injection electrode. Additionally, the ions hit the quadrupole rods due to the low RF amplitude, which according to the Mathieu equations [15] results in unstable ion trajectories.

To reduce such losses and improve the mini-RFQ efficiency, its geometry is modified to accommodate the target plate at the same height as the injection electrode. In order to
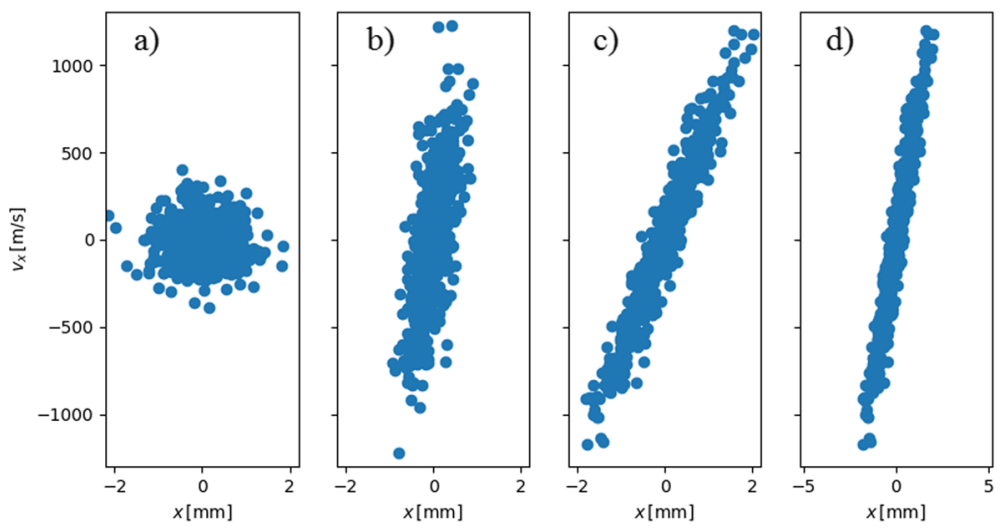

Fig. 7 The phase space of the ions in x-direction at four different time steps corresponding to: a the end of cooling process, $\mathbf{b}$ the ejection from the mini-RFQ, $\mathbf{c}$ the extraction through the first electrode, and $\mathbf{d}$ the detection 

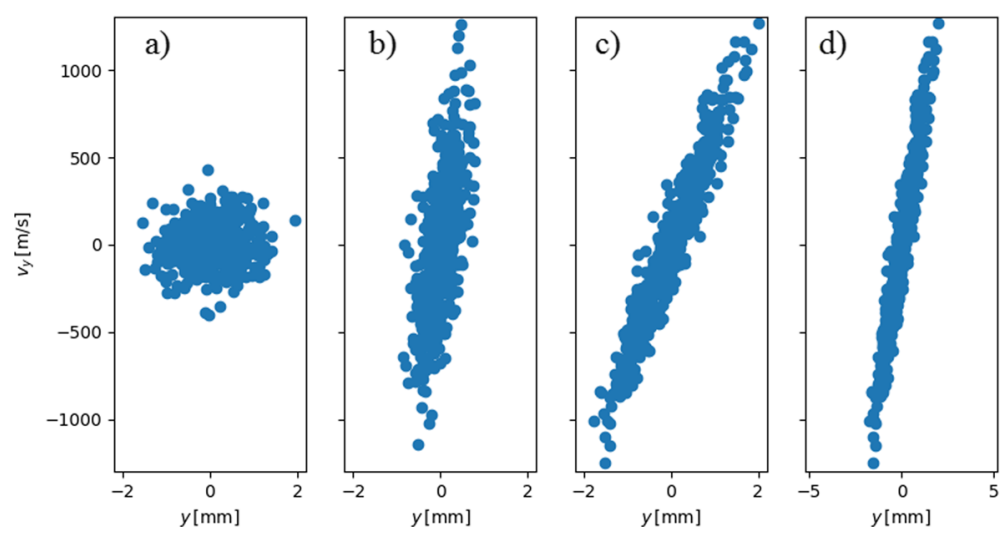

Fig. 8 The phase space of the ions in y-direction at four different time steps corresponding to: a the end of cooling process, $\mathbf{b}$ the ejection from the mini-RFQ, $\mathbf{c}$ the extraction through the first electrode, and $\mathbf{d}$ the detection

operate the mini-RFQ well within the stability region defined by the Mathieu equations for a mass to charge ratio of 193 , an RF amplitude of to $U_{R F}=110 \mathrm{~V}$ was chosen at a frequency of $500 \mathrm{kHz}$. The simulation is extended to the ion transfer through the first extraction electrode. Figure $4 \mathrm{~b}$ and $\mathrm{c}$ and $\mathrm{d}$ show the expected performance with the improved configuration and illustrate the ion cooling, the extraction and the transport, respectively. The positions of the ions are taken at three different time steps. The first time step represents the end of a $2 \mathrm{~ms}$ cooling process through the collisions with helium gas, which is completed without any ion losses. The second time step is taken during the ejection from the mini-RFQ, which occurs without ion losses, as well the third time step, which is taken after transmission of the ions through the ejection electrode and the following electrostatic element, i.e. an electrode in Pierce geometry with a cylindrical channel [16].

The cooling time, i.e. the minimum time needed to thermalize the ions into the minimum of the potential well created inside the mini-RFQ is also studied. Figure 5 shows the trend of the axial and radial kinetic energy of one ion over a cooling period of $2 \mathrm{~ms}$ at a helium gas pressure of 0.01 mbar. Since the mini-RFQ is not vacuum-wise decoupled from the rest of the ion source, its buffer-gas pressure cannot be further increased without deteriorating the good vacuum and hence the ion transmission towards the deflector to the main beamline section. The ion axial motion inside the mini-RFQ is cooled within half a millisecond with a damping constant of $0.16 \mathrm{~ms}$. As the model calculates the deflection angle for ion-atom collisions, the elastic scattering is included: this can result in a very small increase of the axial energy. The radial motion in general is damped in a similar manner, but remains at a radial kinetic energy of about $0.2 \mathrm{eV}$. The higher fluctuations of the radial energy are probably caused by the RF field according to the ion's radial distribution in the radial pseudopotential.

The simulations described above are further extended to the transfer of 500 ions through the extraction electrodes, the quadrupole deflector and the lenses in the high-vacuum regime (see Fig. 2). The DC potential distribution that is applied to the ion optics in order to guide the ions towards the beamline is shown in Fig. 6a where the color code refers to the applied voltage. Figure $6 \mathrm{~b}$ shows the ion trajectories together with their kinetic energy, which are indicated by the color code. By comparing the number of ablated ions with the number of detected ions, a transmission efficiency of $93 \%$ is obtained. 

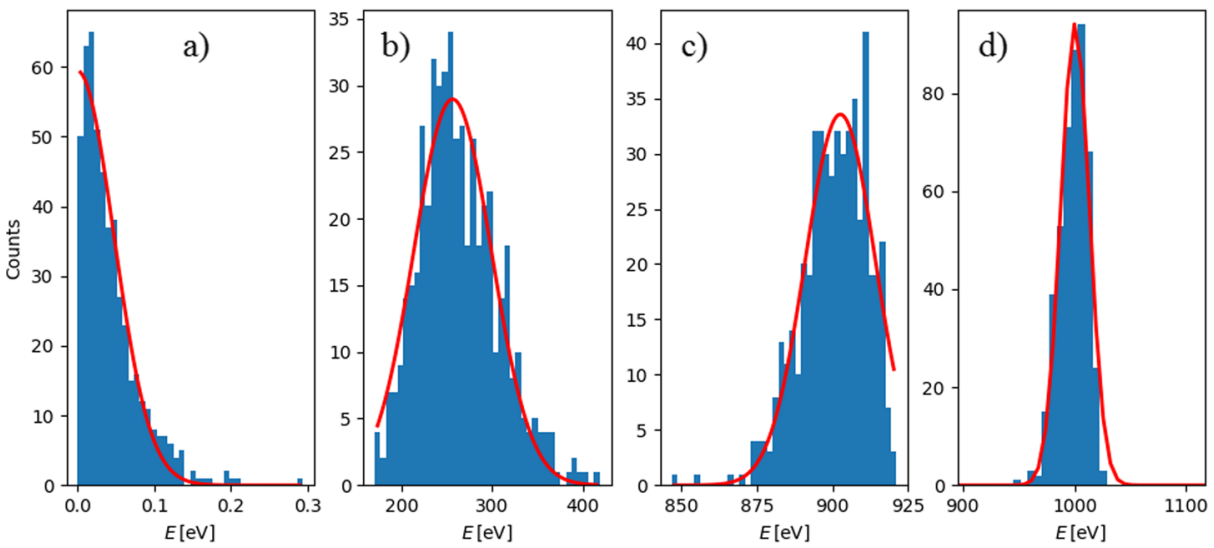

Fig. 9 The energy distribution of the ions at four different time steps corresponding to: a the end of cooling process, $\mathbf{b}$ the ejection from the mini-RFQ, $\mathbf{c}$ the extraction through the first electrode, and $\mathbf{d}$ the detection. The red line represents a fit of a Gaussian to the simulated data points

In Figs. 7 and 8, the phase space diagrams corresponding to the time steps introduced in Fig. $4 b$ and $c$ and $d$ as well as at the position of the detector (shown in Fig. 6b) are displayed. Due to the the conical shape of the mini-RFQ extraction electrode and the Pierce geometry of the following element, the created DC potential distribution is focusing the ion bunch such that the ion trajectories follow straight lines during extraction. Indeed, as shown in Figs. 7b and 8b, during extraction the ions' radial distribution becomes narrower in comparison with their radial distribution right after the cooling process shown in Figs. 7a and $8 \mathrm{a}$. Figures $7 \mathrm{c}$ and $8 \mathrm{c}$ show the phase space of the ions after passing through the aperture of the Pierce-shaped electrode. The final phase space distribution at the position of the detector is shown in Figs. 7d and 8d.

The energy distribution of the ions at the same time steps are presented in Fig. 9. At the end of cooling process the average ion energy is around $0.05 \mathrm{eV}$. A potential difference between the ejection electrode and the first extraction electrode assures the acceleration of the ions towards the beamline and, therefore, shifts the energy distribution to the mean energy of around $256 \mathrm{eV}$ with a FWHM of about $12 \mathrm{eV}$ (see Fig. 9b). The simulation indicates that the bunch length during extraction increases to about $1 \mu \mathrm{s}$. The transport voltage applied to the beamline segments shifts the energy distribution further to the mean energy of $902 \mathrm{eV}$ as shown in Fig. 9c while the energy spread remains similar. Figure 9d shows the final energy distribution registered at the position of the detector with the mean energy of $1000 \mathrm{eV}$ with $13 \mathrm{eV}$ FWHM and the corresponding bunch length of around $1 \mu \mathrm{s}$.

\section{Conclusion and outlook}

An efficient performance of the laser ablation ion source is essential for studying long-lived rare and radioactive isotopes which are available or can be handled only in small quantities. In order to boost the ion production efficiency, a mini RFQ has been implemented for a more efficient capturing of the ablated ions, to decrease the energy spread and reduce the phase space volume of the laser-ablated ions and thus ensure an efficient transport and injection into the traps. 
In this work, simulation studies of the existing setup have been presented. They indicate that ion bunch production can be improved with a different configuration of the target plate, closer to the mini-RFQ injection electrode. The simulated ion bunch production and transmission efficiency for the optimized conditions is $93 \%$. The energy spread of the detected ion bunch is in the order of $13 \mathrm{eV}$ while the time spread is within $1 \mu \mathrm{s}$, which meets the requirements for efficient trapping in the purification trap of SHIPTRAP.

Following the results of the presented simulation studies, a new mini-RFQ with improved geometry has been machined and it is ready for installation into the SHIPTRAP ion source section. At the same time, a new RF inductor (coil) with better impedance matching and thus better amplification power has been implemented in order to allow the use of even higher RF amplitudes for the mini-RFQ rods.

Depending on the mass-to-charge ratio of the ion of interest, either the laser ablation or the surface ion source can be used to provide the most suitable reference ion. In addition, they can also be used to study systematic uncertainties in a mass measurement.

Open Access This article is licensed under a Creative Commons Attribution 4.0 International License, which permits use, sharing, adaptation, distribution and reproduction in any medium or format, as long as you give appropriate credit to the original author(s) and the source, provide a link to the Creative Commons licence, and indicate if changes were made. The images or other third party material in this article are included in the article's Creative Commons licence, unless indicated otherwise in a credit line to the material. If material is not included in the article's Creative Commons licence and your intended use is not permitted by statutory regulation or exceeds the permitted use, you will need to obtain permission directly from the copyright holder. To view a copy of this licence, visit http://creativecommonshorg/licenses/by/4.0/.

\section{References}

1. Blaum, K., Dilling, J., Nörtershäuser, W.: Precision atomic physics techniques for nuclear physics with radioactive beams. Phys. Scr. T152, 014017 (2013). https://doi.org/10.1088/00318949/2013/t152/014017

2. Block, M., Ackermann, D., Blaum, K., Chaudhuri, A., Di, Z., Eliseev, S., Ferrer, R., Habs, D., Herfurth, F., Heßberger, F.P., Hofmann, S., Kluge, H.J., Maero, G., Martín, A., Marx, G., Mazzocco, M., Mukherjee, M., Neumayr, J.B., Plaß, W.R., Quint, W., Rahaman, S., Rauth, C., Rodríguez, D., Scheidenberger, C., Schweikhard, L., Vorobjev, G., Weber, C.: Towards direct mass measurements of nobelium at SHIPTRAP. The European Phys. J. D 45(1), 39 (2007). https://doi.org/10.1140/epjd/e2007-00189-2

3. Block, M., Ackermann, D., Blaum, K., Droese, C., Dworschak, M., Eliseev, S., Fleckenstein, T., Haettner, E., Herfurth, F., Heßberger, F.P., Hofmann, S., Ketelaer, J., Ketter, J., Kluge, H.J., Marx, G., Mazzocco, M., Novikov, Y.N., Plaß, W.R., Popeko, A., Rahaman, S., Rodríguez, D., Scheidenberger, C., Schweikhard, L., Thirolf, P.G., Vorobyev, G.K., Weber, C.: Direct mass measurements above uranium bridge the gap to the island of stability. Nature 463, 785 (2010). https://doi.org/10.1038/nature08774

4. Ramirez, E.M., Ackermann, D., Blaum, K., Block, M., Droese, C., Düllmann, C.E., Dworschak, M., Eibach, M., Eliseev, S., Haettner, E., Herfurth, F., Heßberger, F.P., Hofmann, S., Ketelaer, J., Marx, G., Mazzocco, M., Nesterenko, D., Novikov, Y.N., Plaß, W.R., Rodríguez, D., Scheidenberger, C., Schweikhard, L., Thirolf, P.G., Weber, C.: Direct Mapping of nuclear shell effects in the heaviest elements. Science 337(6099), 1207 (2012). https://doi.org/10.1126/science.1225636

5. Weber, C., Elomaa, V.V., Ferrer, R., Fröhlich, C., Ackermann, D., Äystö, J., Audi, G., Batist, L., Blaum, K., Block, M., Chaudhuri, A., Dworschak, M., Eliseev, S., Eronen, T., Hager, U., Hakala, J., Herfurth, F., Heßberger, F.P., Hofmann, S., Jokinen, A., Kankainen, A., Kluge, H.J., Langanke, K., Martín, A., Martínez-Pinedo, G., Mazzocco, M., Moore, I.D., Neumayr, J.B., Novikov, Y.N., Penttilä, H., Plaß, W.R., Popov, A.V., Rahaman, S., Rauscher, T., Rauth, C., Rissanen, J., Rodríguez, D., Saastamoinen, A., Scheidenberger, C., Schweikhard, L., Seliverstov, D.M., Sonoda, T., Thielemann, F.K., Thirolf, P.G., Vorobjev, G.K.: Mass measurements in the vicinity of the rp-process and the vp-process paths with the Penning trap facilities JYFLTRAP and SHIPTRAP. Phys. Rev. C 78, 054310 (2008). https://doi.org/10.1103/PhysRevC.78.054310 
6. Martın, A., Ackermann, D., Audi, G., Blaum, K., Block, M., Chaudhuri, A., Di, Z., Eliseev, S., Ferrer, R., Habs, D., Herfurth, F., Heßberger, F.P., Hofmann, S., Kluge, H.J., Mazzocco, M., Mukherjee, M., Neumayr, J.B., Novikov, Y., Plaß, W., Rahaman, S., Rauth, C., Rodrıguez, D., Scheidenberger, C., Schweikhard, L., Thirolf, P.G., Vorobjev, G., Weber, C.: Mass measurements of neutron-deficient radionuclides near the end-point of the rp-process with SHIPTRAP. The European Phys. J. A 34(4), 341 (2007). https://doi.org/10.1140/epja/i2007-10520-5

7. Eliseev, S., Blaum, K., Block, M., Chenmarev, S., Dorrer, H., Düllmann, C.E., Enss, C., Filianin, P.E., Gastaldo, L., Goncharov, M., Köster, U., Lautenschläger, F., Novikov, Y.N., Rischka, A., Schüssler, R.X., Schweikhard, L., Türler, A.: Direct measurement of the mass differenc e of ${ }^{163}$ Ho and ${ }^{163}$ Dy solves the $Q$-value puzzle for the neutrino mass determination. Phys. Rev. Lett. 115, 062501 (2015). https://doi.org/10.1103/PhysRevLett.115.062501

8. Chaudhuri, A., Block, M., Eliseev, S., Ferrer, R., Herfurth, F., Martín, A., Marx, G., Mukherjee, M., Rauth, C., Schweikhard, L., Vorobjev, G.: Carbon-cluster mass calibration at SHIPTRAP. The European Phys. J. D 45(1), 47 (2007). https://doi.org/10.1140/epjd/e2007-00001-5

9. Münzenberg, G., Faust, W., Hofmann, S., Armbruster, P., Güttner, K., Ewald, H.: The velocity filter SHIP, a separator of unslowed heavy ion fusion products. Nucl. Inst. Methods 161(1), 65 (1979). https://doi.org/10.1016/0029-554X(79)90362-8

10. Kaleja, O., Andelić, B., Blaum, K., Block, M., Chhetri, P., Droese, C., Düllmann, C., Eibach, M., Eliseev, S., Even, J., Götz, S., Giacoppo, F., Kalantar-Nayestanaki, M., Laatiaoui, M., Ramirez, E.M., Mistry, A., Murböck, T., Raeder, S., Schweikhard, L., Thirolf, P.: The performance of the cryogenic buffer-gas stopping cell of SHIPTRAP. https://doi.org/10.1016/j.nimb.2019.05.009 (2019)

11. Savard, G., Becker, S., Bollen, G., Kluge, H.J., Moore, R., Otto, T., Schweikhard, L., Stolzenberg, H., Wiess, U.: A new cooling technique for heavy ions in a Penning trap. Phys. Lett. A 158(5), 247 (1991). https://doi.org/10.1016/0375-9601(91)91008-2

12. Eliseev, S., Blaum, K., Block, M., Dörr, A., Droese, C., Eronen, T., Goncharov, M., Höcker, M., Ketter, J., Ramirez, E.M., Nesterenko, D.A., Novikov, Y.N., Schweikhard, L.: A phaseimaging technique for cyclotron-frequency measurements. Appl. Phys. B 114(1), 107 (2014). https://doi.org/10.1007/s00340-013-5621-0

13. Schneider, F., Beyer, T., Blaum, K., Block, M., Chenmarev, S., Dorrer, H., Düllmann, C.E., Eberhardt, K., Eibach, M., Eliseev, S., Grund, J., Köster, U., Nagy, S., Novikov, Y.N., Renisch, D., Türler, A., Wendt, K.: Preparatory studies for a high-precision Penning-trap measurement of the ${ }^{163}$ Ho electron capture $Q$-value. The European Phys. J. A 51(7), 89 (2015). https://doi.org/10.1140/epja/i2015-15089-8

14. COMSOL Multiphysics v. 5.4. www.comsol.com

15. March, R., Todd, J.: Quadrupole Ion Trap Mass Spectrometry. Wiley, New York (2007)

16. Pierce, J.R.: Theory and Design of Electron Beams. Van Nostrand, New York (1954)

Publisher's note Springer Nature remains neutral with regard to jurisdictional claims in published maps and institutional affiliations. 